\title{
Negative Appendectomy Rate and Its Correlation with Use of Radiology; a Clinical Audit of Our Department.
}

Aliya Ishaq ${ }^{1,2 *}$,Sameera Naureen ,Yasir Amin ,Jamshaid Hussain Khan ,Atif Latif ,Siyab Anwar and Esaaf Hassan Ghazi.

${ }^{1}$ Department of General Surgery, Dubai Hospital, United Arab Emirates.

${ }^{2}$ Liaquat National Hospital and Medical College, Karachi, Pakistan

*Corresponding author: Aliya Ishaq, Department of General Surgery, Dubai Hospital, United Arab Emirates, Liaquat National Hospital and Medical College, Karachi, Pakistan.

Received Date: May 08, 2021; Accepted Date: May 15, 2021; Published Date: May 29, 2021

Citation: Ishaq A, Naureen S, Amin Y, Jamshaid H Khan, Latif A, Anwar S and Esaaf H Ghazi.(2021) "Negative appendectomy rate and its correlation with use of radiology; A clinical audit of our department”. J. Surg Case Repo and Imag. 4(5); DOI:10.31579/2690-1897/079

Copyright: (C) 2021 Aliya Ishaq, This is an open-access article distributed under the terms of the Creative Commons Attribution License, which permits unrestricted use, distribution, and reproduction in any medium, provided the original author and source are credited.

\begin{abstract}
;
Introduction; Appendectomy is the most common surgical emergency and negative appendectomy is a one of recognized consequence of appendectomy. Recently an increased use of radiology is seen in diagnosing appendicitis and it has significantly decreased the rate of negative appendectomy.

Every effort should be made to establish an exact diagnosis. If, however, this is impossible and a suspicion of appendicitis exists, exploration is mandatory. It is far better to subject a moderate number of patients to a theoretically unnecessary operation than to let one patient suffer perforation.
\end{abstract}

Aim; Recently we have seen an increased use of radiology in our department for diagnosing appendicitis. The idea of conducting this audit was to calculate our negative appendectomy rate by correlating it with use of radiology and to compare it with international figures and to set up guide lines for use of radiology in diagnosing appendicitis on basis of results of our audit .

Methods; Records of all patients who underwent appendectomy in Dubai Hospital, UAE from jan 2018 to jan 2019 were retrospectively analyzed using electronic record system. Clinical diagnosis and radiological findings were compared with histopathology as gold standard for negative appendectomy rate. The sensitivity and specificity of different radiological procedures was calculated as well.

Results; Total 165 patients underwent appendectomy in specified duration. Over all negative appendectomy rate was $17 \%$ with male being $9.7 \%$ and female rate $31 \%$. CTSCAN was found to be $100 \%$ specific and $91.4 \%$ sensitive in diagnosing appendicitis while clinical diagnosis was accurate in $88.5 \%$ cases.

Keywords: radiology; negative appendectomy

\section{Introduction}

Appendicitis is the most common cause of an acute surgical abdomen, with an estimated lifetime prevalence of 7-8 \%. Despite advances in diagnosis and treatment, it is still associated with significant morbidity $(10 \%)$ and mortality (1-5\%) (1). This rapidly progressing inflammatory process requires prompt removal of the appendix to prevent lifethreatening complications such as ruptured appendix and peritonitis and hence accurate and quick diagnosis is important. Traditionally the diagnosis of acute appendicitis is based on clinical features and physical examination .Over the past two decades, the use of dedicated preoperative ultrasonography (US) and computed tomography (CT) techniques for the evaluation of patients clinically suspected of acute appendicitis has led to improved diagnostic accuracy (1)

Negative appendectomy rate, a recognized consequence of appendectomy varies between $6 \%$ to $40 \%$ in the literature. The suggested acceptable rate of negative appendectomy is $20 \%$.
This rate is considered acceptable to avoid missing cases of appendicitis and possible sequela of appendicitis such as perforation, peritonitis, access formation and sepsis, and also to avoid prolonged hospital stay and financial consequences (1).

However, it can further be reduced by utilizing combined clinical assessment with diagnostic modalities.

We did a retrospective audit for negative appendectomy rate of general surgery department Dubai Hospital, UAE for a duration of one year by correlating the clinical diagnosis of appendicitis with histopathology being gold standard. We also correlated histopathological diagnosis with radiological diagnosis and hence calculated specificity and sensitivity of different radiological procedures in diagnosing appendicitis.

\section{Materials and Methods}

Setting: Department of General Surgery Dubai Hospital,UAE 


\section{Duration of Study: FROM JAN 2018 TO Jan 2019}

Sample Size: total 165 patients who underwent appendectomy during the specified duration were included in audit .

Sampling Technique: continuous sampling

\section{Data Collection:}

Medical records of all the patients were reviewed retrospectively using electronic medical records used in our hospital from jan 2018 to jan 2019.

\section{Data Analysis:}

All analysis will be conducted by using the Statistical package for social sciences (SPSS) version 24. p Value is used for changes in quantitative viable for significant changes and numbers and percentage are used for descriptive variables.

\section{Results}

Total 165 appendectomies were performed between Jan 2018 till Jan 2019.

Mean age was 23 years.

For sex data;

\section{Frequency Table}

\begin{tabular}{|r|c|r|r|r|r|}
\hline \multicolumn{2}{|c|}{} & Frequency & Percent & Valid Percent & $\begin{array}{c}\text { Cumulative } \\
\text { Percent }\end{array}$ \\
\hline \multirow{3}{*}{ Valid } & 1 & 107 & 64.8 & 64.8 & 64.8 \\
\cline { 2 - 6 } & 2 & 58 & 35.2 & 35.2 & 100.0 \\
\cline { 2 - 6 } & Total & 165 & 100.0 & 100.0 & \\
\hline
\end{tabular}

Male patients in audit $107 / 165-64.8 \%$

Female patients in audit 58/165-35.2\%

Histopathological findings;

They were acute inflammation, acute supportive appendicitis, Tran's mural inflammation of appendix with or without fecalith and gangrenous perforated appendix.

8 cases of fibrous obliteration of lumen of appendix with neuroma of tip without inflammation reported

\begin{tabular}{|c|l|r|r|r|r|}
\hline \multicolumn{2}{|c|}{} & Frequency & Percent & Valid Percent & \multicolumn{1}{c|}{$\begin{array}{c}\text { Cumulative } \\
\text { Percent }\end{array}$} \\
\hline \multirow{3}{*}{ Valid } & 1 & 137 & 83.0 & 83.0 & 83.0 \\
\cline { 2 - 6 } & 2 & 28 & 17.0 & 17.0 & 100.0 \\
\cline { 2 - 6 } & Total & 165 & 100.0 & 100.0 & \\
\hline
\end{tabular}

137 out of 165 showed that its appendicitis ie $83 \%$

Ct scan abdomen and pelvis was done in $63.0 \%$ patients

28/165 showed negative appendectomy ie $-17 \%$

Us abdomen done in $5.4 \%$ cases

Negative appendectomy rate is $17 \%$

Imaging used over all in $68.4 \%$ cases

Ct scan findings;

31.55 cases had clinical diagnosis.

\begin{tabular}{|r|l|r|r|r|r|}
\hline \multicolumn{2}{|c|}{ ct scan findings } \\
\hline \multirow{3}{*}{ Valid } & Frequency & Percent & Valid Percent & $\begin{array}{c}\text { Cumulative } \\
\text { Percent }\end{array}$ \\
\cline { 2 - 6 } & 1 & 93 & 56.4 & 56.4 & 56.4 \\
\cline { 2 - 6 } & 2 & 11 & 6.7 & 6.7 & 63.0 \\
\cline { 2 - 6 } & 3 & 4 & 2.4 & 2.4 & 65.5 \\
\cline { 2 - 6 } & 4 & 5 & 3.0 & 3.0 & 68.5 \\
\cline { 2 - 6 } & 5 & 52 & 31.5 & 31.5 & 100.0 \\
\cline { 2 - 6 } & Total & 165 & 100.0 & 100.0 & \\
\hline
\end{tabular}

1. Ct scan done and is positive for appendicitis - 93/165-56.4\%

2. $\mathrm{Ct}$ scan done and is negative ie normal appendix $-11 / 165-6.7 \%$

3. Us done and showed appendicitis $-4 / 165-2.4 \%$

4. Us done and showed normal appendix $-5 / 165-3 \%$

5. No image done /clinical diagnosis 52/165 -31.5\% 


\begin{tabular}{|c|c|c|c|c|c|}
\hline \multicolumn{6}{|c|}{ Ct scan findings $*$ Histopathology reports Cross tabulation } \\
\hline & & & \multicolumn{2}{|c|}{ Histopathology reports } & \multirow[t]{2}{*}{ Total } \\
\hline & & & 1 & 2 & \\
\hline \multirow[t]{5}{*}{ ct scan findings } & \multirow[t]{2}{*}{1} & Count & 85 & 8 & 93 \\
\hline & & $\%$ within ct scan findings & $91.4 \%$ & $8.6 \%$ & $100.0 \%$ \\
\hline & \multirow[t]{2}{*}{2} & Count & 0 & 11 & 11 \\
\hline & & $\%$ within ct scan findings & $0.0 \%$ & $100.0 \%$ & $100.0 \%$ \\
\hline & & Count & 2 & 2 & 4 \\
\hline
\end{tabular}

\begin{tabular}{|l|l|r|r|r|r|}
\hline \multirow{3}{*}{4} & \% within ct scan findings & $50.0 \%$ & $50.0 \%$ & $100.0 \%$ & \\
\hline \multirow{2}{*}{5} & Count & 4 & 1 & 5 & \\
\cline { 2 - 6 } & \% within ct scan findings & $80.0 \%$ & $20.0 \%$ & $100.0 \%$ & \\
\hline \multirow{2}{*}{ Total } & Count & 46 & 6 & 52 & \\
\cline { 2 - 6 } & $\%$ within ct scan findings & $88.5 \%$ & $11.5 \%$ & $100.0 \%$ & \\
\hline
\end{tabular}

1. $91.4 \%$ of ct diagnosed patients have histologically proven appendicitis while $8.6 \%$ of ct diagnosed patients have negative appendectomy.

2. All patients who had normal appendix on ct had normal appendix on histopathology as well. Sensitivity of ct scan is $91.4 \%$ while specificity is $100 \%$.

3. All patients who had us done and it showed appendicitis out of all these patients $50 \%$ had histologically proved appendicitis and 50
$\%$ had histologically negative appendix, ie sensitivity of us is $50 \%$ in detecting appendicitis.

4. Patients who's ultra sound showed normal appendix $80 \%$ of these patients had appendicitis on histopathology and $20 \%$ had normal appendix on histopathology .specificity of us is $20 \%$.

5. Patients with clinical diagnosis of appendicitis $88.5 \%$ of these patients had appendicitis on histopathology and $17 \%$ had normal appendix on histopathology.

\begin{tabular}{|c|c|c|c|c|c|}
\hline \multicolumn{6}{|c|}{ Sex * Histopathology reports Cross tabulation } \\
\hline & & & \multicolumn{2}{|c|}{ Histopathology reports } & \multirow[t]{2}{*}{ Total } \\
\hline & & & 1 & 2 & \\
\hline \multirow[t]{4}{*}{ Sex } & \multirow[t]{2}{*}{1} & Count & 97 & 10 & 107 \\
\hline & & $\%$ within Sex & $90.7 \%$ & $9.3 \%$ & $100.0 \%$ \\
\hline & \multirow[t]{2}{*}{2} & Count & 40 & 18 & 58 \\
\hline & & $\%$ within Sex & $69.0 \%$ & $31.0 \%$ & $100.0 \%$ \\
\hline \multirow{2}{*}{\multicolumn{2}{|c|}{ Total }} & Count & 137 & 28 & 165 \\
\hline & & $\%$ within Sex & $83.0 \%$ & $17.0 \%$ & $100.0 \%$ \\
\hline
\end{tabular}

1. Negative appendectomy rate in male patients was $9.7 \%$ while in female patients it was $31 \%$.

\section{Discussion}

Several recent papers have cited a declining negative appendectomy rate( NAR), including several large database studies and meta analyses with NARs as low as $6-8 \%$ and single institution studies with NARs as low as $1.7-7 \%$, coinciding with the increased use of computed tomography (CT) and laparoscopyi. While CT is often credited with lowering the NAR, a definitive causal relationship has not been established and lingering questions about proper usage remain. Routine CT is unnecessary for male patients with clinical diagnosis of appendicitis. Mild appendicitis may resolve without surgery and CT may contribute to unnecessary surgery (2).

.Over the past two decades, the use of dedicated pre-operative ultrasonography (US) and computed tomography (CT) techniques for the evaluation of patients clinically suspected of acute appendicitis has led to improved diagnostic accuracy ${ }^{\mathrm{ii}}$. In light of this, in 2010 the Dutch College of Surgeons introduced a guideline entitled "diagnostics and treatment in acute appendicitis" with recommendations concerning pre-operative imaging in the diagnosis and treatment of acute appendicitis. The guideline states that in every patient with clinically suspected acute appendicitis an ultrasonography or CT scan is advised to confirm diagnosis before surgery (3).

When compared with patients with appendicitis, negative appendectomy was associated with a significantly longer length of stay (5.8 vs. 3.6 days, $\mathrm{P}<0.001)$, infectious complications rate $(2.6 \%$ vs. $1.8 \%, \mathrm{P}<0.001)$ case fatality rate $(1.5 \%$ vs. $0.2 \%, \mathrm{P}<0.001)$ and total charge-admission $(\$ 18,780$ vs. $10,584, \mathrm{P}<0.001)$. An estimated $\$ 741.5$ million in total hospital charges resulted from admissions in which a negative appendectomy was performed (3).

Higher NAR in the female sex compared to the male sex have been reported by multiple studies. Seetha et al(3) in a 10-year review of a nationally representative sample of 475,651 cases of appendectomy reported that women accounted for $71.6 \%$ of the negative appendectomies. This is consistent with the findings of this study in which females accounted for approximately $60 \%$ of the negative appendectomies. Reasons adduced for this observation includes the gynecological conditions that could mimic the presentation of acute appendicitis. Ovarian cysts, leiomyoma, endometriosis, benign ovarian neoplasms, malignant ovarian disease, pelvic adhesions have been reportedly misdiagnosed as acute appendicitis in women (10) (11). 
Our audit showed an overall negative appendectomy rate of $17 \%$ while it is $9.7 \%$ in male and $31 \%$ in female patients. Imaging was used over all in $68.4 \%$ cases while $31.55 \%$ cases had clinical diagnosis. CT SCAN was done in $63 \%$ cases while ultrasound was done in only $5.4 \%$ cases.

Moreover our audit showed that the type of CT SCAN used was not consistent and following types of different CT scans were used

CT kub 20/93, 21.5\%

CT kub followed by ct with contrast $9 / 93,5.4 \%$

CT scan abdomen plain 12/93,7.2\%

CT scan abdomen with iv contrast 49/93,52.6\%

CT scan abdomen with iv and oral contrast 9/93,9.6\%

Our results show that $\mathrm{Ct}$ scan abdomen has $100 \%$ specificity in diagnosing appendicitis while sensitivity of $\mathrm{Ct}$ scan is $91.4 \%$ at the same time clinical diagnosis alone without help of imaging diagnosed $88 \%$ cases of appendicitis.

We used imaging in all female patients and male patients above 40 years. Imaging was also used in patients where history was not clear or history was 3 days or more to rule out appendicular mass.

Clinical diagnosis was made on basis of history and Alvarado score.

The results on basis of this audit cannot be generalized as the number of patients are very small and there was no consistent guidelines for use of imaging (Ct scan /ultrasound abdomen) further more even the $\mathrm{Ct}$ scan abdomen was not done with one protocol some patients have plain some had with contrast so the results are biased

The suggestion is as follows;

\section{CT scan is better than us in diagnosing appendicitis.}

Use of us should be restricted where CT scan is a contraindication as sensitivity and specificity is low and should be combined with clinical diagnosis.

\section{Clinical diagnosis should be combined with use of CT scan} whenever indicated as follows;

a. All female patients.

b. Patients with h/o appendicitis with more than 3 days duration.

c. Patients with nonspecific history.

d. All patients with age above 40 should have CT SCAN abdomen in case of clinical suspected appendicitis, to rule out malignancy and other rare pathologies.

Clinical and laboratory parameters not matching.

Complicated cases.

Also we agreed with our radiology department to go for ct scan abdomen with oral and IV contrast if needed for diagnosis of acute appendicitis, although nowadays FACT is used to diagnose but as for our institute most of radiologist agreed that in thin patients non contrast ct scan is not help full and can give false information.
Also as far as histopathology is concerned most of our colleagues said that it should be double checked with 2 histopathologist but keeping in mind the frequent cases of appendicitis coming to emergency and as a volume overload it is difficult to implement that outside research area.

On the basis of results of this audit local guide lines for us of CT SCAN abdomen are made for our department saying that appendicitis should be a clinical diagnosis and where there is doubt about the diagnosis ( conditions described above ) radiology help should be taken .Moreover all female patients should have radiology prior to subjecting any patient for appendectomy. Whenever CT SCAN is needed it should be done with both IV and oral contrast (although many people will not agree for it especially with advent of FACT, but we will be able to give an opinion after re-audit). Based on these implementation of these guidelines the department will recon duct the audit after 2 years and results will be evaluated again. Furthermore, another prospective research study can be conducted to look for sensitivity and specificity of CT SCAN in diagnosing acute appendicitis and results can be implemented then.

\section{References:}

1. Yara F et al (2018). Negative Appendectomy Rate and Risk Factors That Influence Improper Diagnosis at King Abdul-Aziz University Hospital Mater Sociome; 30(3): 215-220

2. Kathryn T et al. (2016). Negative Appendectomy Rates Do Not Increase with Reduced Computed Tomography Use in Pediatric Appendicitis. JACS -223(4);158

3. Narayanan A et al. (2015). What is negative about negative appendectomy rates? An experience from a district general hospital. Int Surg J; 2(2):161-164

4. JG Mariadason et al. (2012). Negative appendectomy rate as a quality metric in the management of appendicitis: impact of computed tomography, Alvarado score and the definition of negative appendectomy. Ann R Coll Surg Engl; 94: 395-401

5. Gomes et al. (2015). Acute appendicitis: proposal of a new comprehensive grading system based on clinical, imaging and laparoscopic findings. World Journal of Emergency Surgery; 10:60

6. Kathryn T et al. (2016). Negative Appendectomy Rates Do Not Increase with Reduced Computed Tomography Use in Pediatric Appendicitis. JACS; 223(4);158

7. P. A. Boonstra, R. N. van Veen, H. B. A. C. (2015). Less negative appendectomies due to imaging in patients with suspected appendicitis. Stockmann Surg Endosc; 29:2365-2370

8. Marudanayagam R, Williams GT, Rees BI. (2006). Review of the pathological results of 2660 appendectomy specimens. $J$ Gastroenterol; 41:745-749.

9. Seetahal etal. (2011). Negative appendectomy a 10 year review of a nationally representative sample. Am J Surg; 201(4);433-437.

10. Nicolo Tamini, Letizia Santurro, Maria Francesca Chiappetta, Ilaria Gattuso, Consuelo Barbieri et.al (2020) Morbidity after negative appendectomy: a single-centre experience on 627 cases. European Journal of Trauma and Emergency Surgery; 46:859864

11. Yara F. Alhamdani, Hisham A. Rizk, Mohammed R. Algethami, Asma M. Algarawi, Roia H. Albadawi. (2018). Negative Appendectomy Rate and Risk Factors That Influence Improper Diagnosis at King Abdulaziz University Hospital. Mater Sociomed; 30(3): 215-220 